\title{
SARS-CoV-2 infection in a 76-year-old man with initially negative nasopharyngeal swabs
}

\author{
Divjot S. Kumar MD, Siobhán B. O’Neill MD PhD, James C. Johnston MD MPH, Jennifer M. Grant MD, \\ David D. Sweet MD
}

Cite as: CMAJ 2020 May 19;192:E546-9. doi: 10.1503/cmaj.200641; early-released April 24, 2020

A

76-year-old man presented to a quaternary care hospital with a 6-day history of respiratory symptoms, fatigue and a fainting episode. The patient had a resolving postinfectious dry cough from an upper respiratory infection that occurred 3 months before presentation. He had hypertension, gastritis, diabetes, sciatica, a remote 30 pack-year smoking history and no history of recreational drug use. His medications included metformin and rosuvastatin. He worked as a professor and attended language classes.

In early March, our patient was informed of a potential classroom exposure to coronavirus disease 2019 (COVID-19). One week after this exposure, he had an outpatient nasopharyngeal swab for severe acute respiratory syndrome coronavirus 2 (SARSCoV-2) after 2 days of worsening cough. The results were negative for both the envelope and RNA-dependent RNA polymerase genes, which are common targets for polymerase chain reaction (PCR) amplification of SARS-CoV-2. A worsening dry cough, fatigue, exertional dyspnea, fevers, low appetite and diarrhea developed over the next 4 days. He presented to the emergency department 4 days after his first swab for SARS-CoV-2 (11 d after his potential exposure).

The patient was admitted to hospital and placed under droplet and contact precautions. His initial vital signs included a body temperature of $38.6^{\circ} \mathrm{C}$, blood pressure of $98 / 55 \mathrm{~mm} \mathrm{Hg}$ with an orthostatic drop, pulse 94 beats/min, and a respiratory rate of 18 breaths/ min with an oxygen saturation of $96 \%$ on room air. A physical examination showed flat neck veins, and he had mild inspiratory bibasilar crackles. Bloodwork showed lymphopenia, but electrolytes and hepatic and renal function tests were normal. Chest radiography showed ill-defined right basal airspace opacification.

We started treatment with crystalloids administered intravenously and ceftriaxone and azithromycin for pneumonia. Blood and stool culture tests performed on admission were negative; influenza $A$, influenza $B$ and respiratory syncytial virus were not present; and a repeat nasopharyngeal swab for SARSCoV-2 on admission also returned a negative result.

On day 3 after admission, our patient became hypoxemic and he required $2 \mathrm{~L} / \mathrm{min}$ of oxygen. Repeat radiography of the chest showed

\section{KEY POINTS}

- The sensitivity of testing using real-time reverse transcriptase polymerase chain reaction for nasopharyngeal or oropharyngeal swabs for severe acute respiratory syndrome coronavirus 2 (SARS-CoV-2) is affected by sampling technique and timing within the disease course.

- Studies have shown negative SARS-CoV-2 nasopharyngeal test results for patients with a clinical course suggestive of coronavirus disease 2019 , who have subsequently tested positive.

- The possibility of nosocomial acquisition and transmission of SARS-CoV-2 from asymptomatic health care workers supports the use of universal precautions in patient care.

new bilateral, ill-defined patchy opacities. Results for an extended viral panel, legionella urinary antigen and a third nasopharyngeal swab for SARS-CoV-2 were negative. Consequently, we stopped droplet and contact precautions on day 3 of admission. A computed tomography (CT) scan of the chest showed bilateral ground glass opacification and septal lines (Figure 1). This was consistent with atypical bacterial or viral infection, and our differential diagnosis included edema, hemorrhage, drug reaction and connective tissue disease.

On day 4 of admission, our patient's hypoxemia worsened and he required $5 \mathrm{~L} / \mathrm{min}$ of oxygen. We consulted our respirology department. Given his history of progressive symptoms, persistent lymphopenia and potential exposure to a student with COVID-19, we were advised to restart droplet and contact precautions. ${ }^{1}$ We stopped the previous antibiotic regimen and started broader antibiotic treatment (piperacillin/tazobactam and vancomycin). We also started empiric treatment with methylprednisolone (125 mg administered intravenously once daily) for possible organizing pneumonia. Results were negative for sputum cultures and a fourth nasopharyngeal swab for SARSCoV-2 conducted on day 4 of admission. We transferred the patient to the intensive care unit (ICU), where he was cared for in a negative pressure room. Diuresis was attempted and the 
patient was provided with fraction of inspired oxygen $\left(\mathrm{FiO}_{2}\right)$ at $60 \%$ via a high-flow nasal canula.

By day 5 of admission, his $\mathrm{FiO}_{2}$ requirements had increased to $90 \%$. He was intubated and ventilated using a lung protective strategy. The dosage of methylprednisolone was increased to $125 \mathrm{mg}$ administered intravenously every 6 hours. Results for tests for connective tissue disease, anti-glomerular basement membrane antibody disease, hepatitis and HIV were negative.

Bronchoscopy on day 6 of admission showed bloody returns on sequential bronchoalveolar lavage. Real-time reverse transcriptase
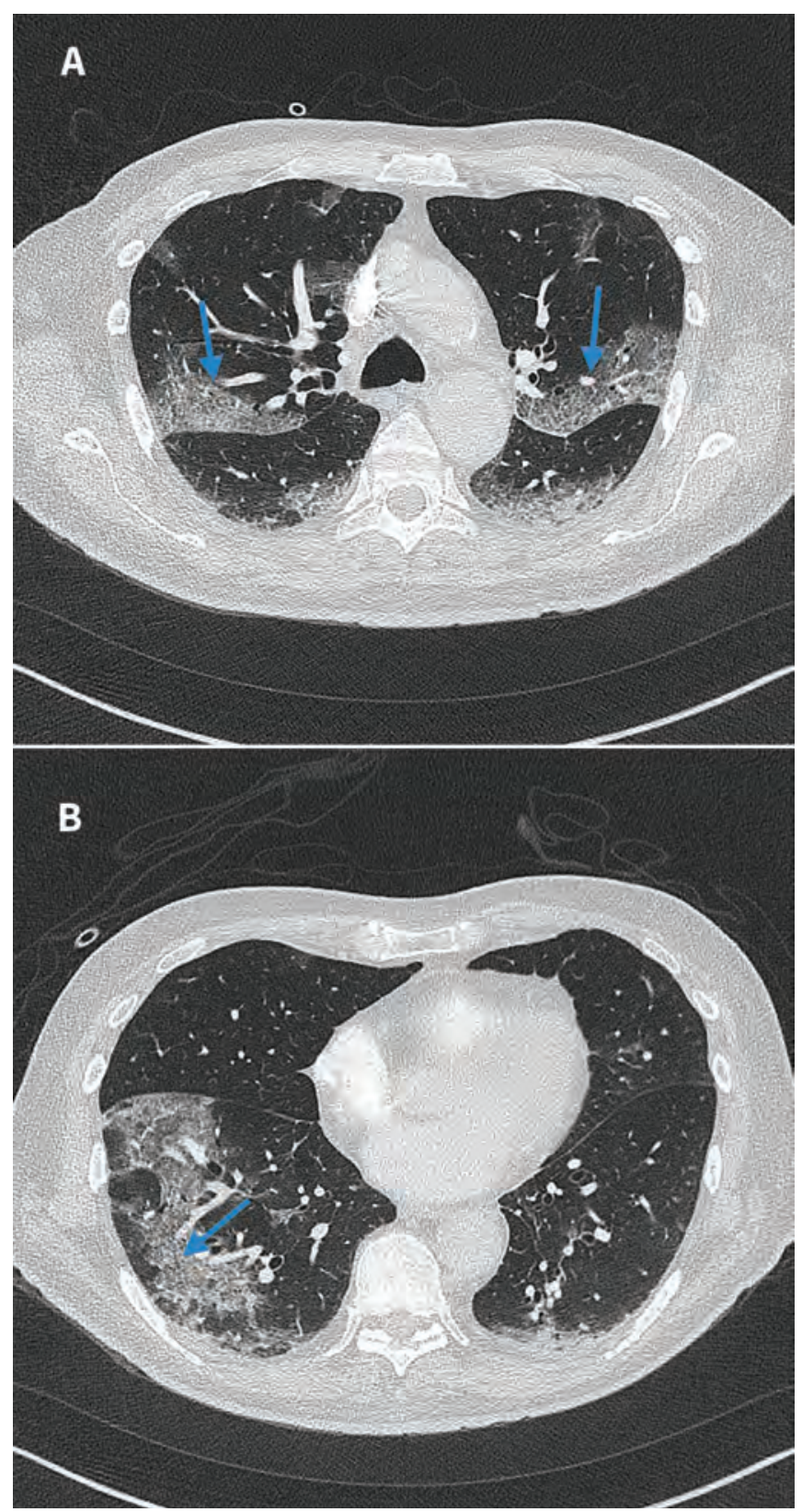

Figure 1: $(A)$ and (B) Computed tomography images of the chest (taken on day 3 of admission to hospital) of a 76-year-old man with coronavirus disease 2019 (COVID-19) and negative results for nasopharyngeal swabs. Bilateral peripheral ground glass opacification with areas of visible septal lines constituting crazy-paving are visible (blue arrows). This is typical of COVID-19 appearance as per the Radiological Society of North America Expert Consensus Statement. ${ }^{1}$
PCR (RT-PCR) testing using samples of the bronchoalveolar lavage and brushings showed a positive result for SARS-CoV-2. On day 7 of admission, results were positive for SARS-CoV-2 for tests of a nasopharyngeal swab and tracheal aspirate. Our patient received supportive treatment and was extubated on day 10 . We reduced his prednisone to $50 \mathrm{mg} / \mathrm{d}$, which we tapered by $5 \mathrm{mg}$ every 3 days. Repeat CT on day 15 of admission (Figure 2) showed segmental pulmonary embolism and evolving lung infiltrates in keeping with an organizing pneumonia pattern. We started therapeutic anticoagulation and discharged the patient home on day 19 after admission.

\section{Discussion}

The early identification of patients with COVID-19 is crucial to initiating appropriate isolation and management. Our case illustrates some diagnostic challenges for COVID-19 and the resulting implications for infection management, including the control of nosocomial transmission.

Although RT-PCR testing for SARS-CoV-2 is the current standard for diagnosis of COVID-19, the sensitivity of nasopharyngeal swabs has been reported to vary, ranging from $42 \%$ to $71 \% .^{1,2}$ In a recent case series of patients with high probability of COVID-19 based on clinical presentation and imaging, only 9 of 19 patients $(47 \%)$ had a positive result for oropharyngeal testing using RTPCR. ${ }^{3}$ Sensitivity of SARS-CoV-2 swabs is thought to be affected by sampling technique and timing within the clinical course of COVID-19. ${ }^{2-4}$ Furthermore, findings from chest CT may precede positive results for RT-PCR tests. ${ }^{1,2} \mathrm{~A}$ recent case of a 28 -year-old Thai man with cough reported 2 negative results for nasopharyngeal swabs by day 5 of admission to hospital, yet subsequent bronchoscopy performed on day 8 obtained bronchoalveolar lavage fluid that tested positive for SARS-CoV-2 using RT-PCR. ${ }^{4}$ Such discrepancies may relate to a prolonged incubation period affecting sensitivity of the pharyngeal swabs. An in vitro study conducted during the SARS outbreak showed that the functional receptor of SARS-CoV was angiotensin-converting enzyme 2 (ACE2). ${ }^{5}$ The minimal expression of ACE2 in the posterior pharynx compared with the lower respiratory tract may account for differences in viral loads by anatomic site, reducing the sensitivity of nasopharyngeal swabs compared with deeper respiratory sampling. ${ }^{4,5}$ The genomic similarity of SARS-CoV and SARS-CoV-2 has led some researchers to hypothesize that similar mechanisms may apply to the current SARS-CoV-2 replication cycle. ${ }^{6}$

The importance of understanding diagnostic testing in the context of the clinical course of COVID-19 is further highlighted by a recent study that assessed viral loads from different anatomic sites and found that SARS-CoV-2 RNA concentrations in pharyngeal swabs were already declining at the time of first clinical presentation, whereas sputum RNA viral concentrations declined more slowly. ${ }^{7}$ The authors of this study noted active pharyngeal viral shedding in mild disease with increasing lower respiratory tract involvement later in the disease trajectory. A recent guideline suggests use of deep respiratory sampling when possible over nasopharyngeal swabs to help improve the accuracy of RT-PCR testing. ${ }^{8}$ Unfortunately, lower respiratory tract 

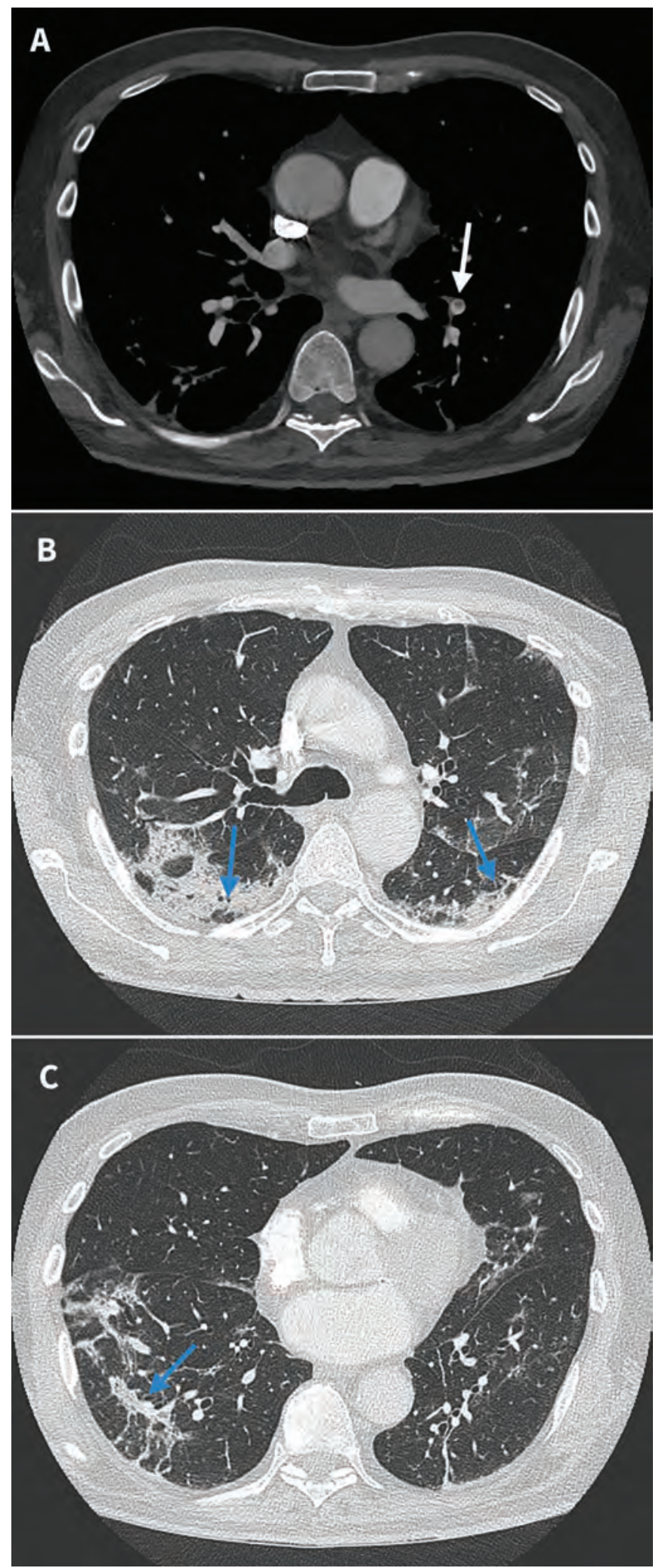

Figure 2: Computed tomography images of the chest taken on day 15 of admission to hospital. (A) Segmental nonocclusive pulmonary embolus in the left lower lobe (white arrow). (B) and (C) Improved ground glass and septal thickening; bilateral peripheral peribronchovascular and perilobular consolidation with mild associated volume loss and parenchymal distortion are visible, in keeping with an evolving organizing pneumonia reaction pattern (blue arrows). samples cannot be readily obtained in all patients suspected of having COVID-19. Bronchoscopy provides access to the lower respiratory tract, but it is an aerosol-generating procedure that can put health care workers at risk of exposure to the virus.

It is possible that our patient was exposed to SARS-CoV-2 in hospital. This supposes he incurred hypoxemic respiratory failure secondary to organizing pneumonia or alveolitis from a cause other than SARS-CoV-2. His initial nasopharyngeal swabs in hospital may have been truly negative, with subsequent bronchoscopy results reflecting in-hospital SARS-CoV-2 acquisition. In addition, the incubation period for COVID-19 is 2-11 days (mean $5 \mathrm{~d}$ ), compatible with a possible SARS-CoV-2 exposure during the brief time he was not in isolation. ${ }^{9}$ The possibility of nosocomial SARS-CoV-2 spread is increasingly recognized; although widespread testing of asymptomatic health care workers is not suggested at present, transmission of SARS-CoV-2 from asymptomatic carriers has been documented. ${ }^{10}$ To reduce transmission risk, the Vancouver General Hospital has a policy of universal masking and eye shielding for all health care providers involved in direct patient care. With the possibility of nosocomial transmission, adherence to strict precautions and good hygiene for asymptomatic health care workers is crucial.

Routine use of corticosteroid therapy in the treatment of COVID-19 is currently not recommended unless other indications exist. ${ }^{8}$ For patients with COVID-19 and acute respiratory distress syndrome (ARDS), however, the use of steroids remains controversial. The Surviving Sepsis Campaign guideline provides a weak recommendation favouring steroid use in patients with COVID-19 and ARDS. ${ }^{8}$ In our case, it is possible that initiation of high-dose corticosteroids led to accelerated viral replication and a positive result for SARS-CoV-2 testing 3 days later. This issue was explored during the previous SARS outbreak where early corticosteroid use appeared to result in higher plasma viral loads and delayed viral clearance. ${ }^{11}$ Given our patient's rapid recovery in the ICU while receiving steroid therapy, we continued corticosteroids with a rapid taper in an effort to mitigate prolonged viral shedding.

\section{Conclusion}

Testing of nasopharyngeal swabs for SARS-CoV-2 using RT-PCR should be interpreted in the context of clinical signs and symptoms. If clinical suspicion of infection associated with COVID-19 remains after an initially negative nasopharyngeal swab, we urge clinicians to continue isolation procedures to prevent nosocomial infection.

\section{References}

1. Simpson S, Kay FU, Abbara S, et al. Radiological Society of North America Expert Consensus Statement on Reporting Chest CT Findings Related to COVID-19. Endorsed by the Society of Thoracic Radiology, the American College of Radiology, and RSNA. Radiology: Cardiothoracic Imaging 2020 Mar. 25;2:e200152. doi: $10.1148 /$ ryct.2020200152.

2. Ai T, Yang Z, Hou H, et al. Correlation of chest CT and RT-PCR testing in coronavirus disease 2019 (COVID-19) in China: a report of 1014 cases. Radiology 2020 Feb. 26 [Epub ahead of print]. 200642. doi: 10.1148/radiol.2020200642.

3. Xie C, Jiang L, Huang G, et al. Comparison of different samples for 2019 novel coronavirus detection by nucleic acid amplification tests. Int J Infect Dis 2020;93:264-7.

4. Winichakoon P, Chaiwarith R, Liwsrisakun C, et al. Negative nasopharyngeal and oropharyngeal swab does not rule put COVID-19. J Clin Microbiol 2020 Feb. 26 [Epub ahead of print]. pii: JCM.00297-20. doi: 10.1128/JCM.00297-20. 
5. Li W, Moore MJ, Vasilieva N, et al. Angiotensin-converting enzyme 2 is a functional receptor for the SARS coronavirus. Nature 2003;426:450-4.

6. Vaduganathan $M$, Vardeny $\mathrm{O}$, Michel $\mathrm{T}$, et al. Renin-angiotensin-aldosterone system inhibitors in patients with COVID-19. NEJM 2020 Mar. 30 [Epub ahead of print]. doi: 10.1056/NEJMsr2005760.

7. Woelfel R, Corman VM, Guggemos W, et al. Clinical presentation and virological assessment of hospitalized cases of coronavirus disease 2019 in a travel-associated transmission cluster. medRxiv 2020 Mar 8. doi: 10.1101/2020.03.05.20030502.

8. Alhazzani W, Møller MH, Arabi YM, et al. Surviving sepsis campaign: guidelines on the management of critically ill adults with coronavirus disease 2019 (COVID-19). Intensive Care Med 2020 Mar. 28 [Epub ahead of print]. doi: 10.1007/s00134-020-06022-5.

9. Backer JA, Klinkenberg D, Wallinga J. Incubation period of 2019 novel coronavirus (2019-nCoV) infections among travellers from Wuhan, China, 20-28 January 2020 [rapid communication]. Euro Surveill 2020 Feb. 6[Epub ahead of print];25. doi: 10.2807/1560-7917.ES.2020.25.5.2000062.

10. Klompas M, Morris CA, Sinclair J, et al. Universal masking in hospitals in the COVID-19 era. NEngl J Med 2020 Apr. 1 [Epub ahead of print]. doi: 10.1056/NEJMp2006372.

11. Lee N, Allen Chan KC, Hui DS, et al. Effects of early corticosteroid treatment on plasma SARS-associated coronavirus RNA concentrations in adult patients. J Clin Virol 2004;31:304-9.

\section{Competing interests: None declared.}

This article has been peer reviewed.

The authors have obtained patient consent.

Affiliations: Divisions of Respiratory Medicine (Kumar, Johnston), Infectious Diseases (Grant) and Critical Care Medicine (Sweet), Department of Radiology (O'Neill), Faculty of Medicine, University of British Columbia, Vancouver, BC

Contributors: Divjot Kumar wrote the first draft of the manuscript. All of the authors equally contributed equally to the conception of the manuscript, reviewed it critically for important intellectual content, gave final approval of the version to be published and agreed to be accountable for all aspects of the work.

Acknowledgement: The authors thank Dr. Tharwat Fera and Dr. Robert Reynolds for their help with the management of this case.

Correspondence to: Divjot Kumar, kumardivjot@gmail.com 